\title{
Inactivation of RASSF1A tumor suppressor gene by aberrant promoter hypermethylation in human pituitary adenomas
}

\author{
Zhi Rong Qian ${ }^{1,2}$, Toshiaki Sano ${ }^{1}$, Katsuhiko Yoshimoto ${ }^{3}$, Shozo Yamada ${ }^{4}$, Akira Ishizuka ${ }^{5}$, \\ Noriko Mizusawa ${ }^{3}$, Hidehisa Horiguchi ${ }^{1}$, Mitsuyoshi Hirokawa ${ }^{1}$ and Sylvia L Asa ${ }^{6}$ \\ ${ }^{1}$ Department of Pathology, Institute of Health Biosciences, The University of Tokushima Graduate School, \\ Tokushima, Japan; ${ }^{2}$ Satellite Venture Business Laboratory, The University of Tokushima, Tokushima, Japan; \\ ${ }^{3}$ Department of Medical Pharmacology, Institute of Health Biosciences, The University of Tokushima, \\ Tokushima, Japan; ${ }^{4}$ Department of Neurosurgery, Toranomon Hospital, Tokyo, Japan; ${ }^{5}$ Institute for Genome \\ Research, The University of Tokushima, Tokushima, Japan and ${ }^{6}$ Department of Laboratory Medicine and \\ Pathobiology, University of Toronto, Toronto, Ontario, Canada
}

\begin{abstract}
Aberrant promoter methylation and resultant silencing of several tumor suppressor genes play an important role in the pathogenesis of many tumor types. The human Ras association domain family 1A gene (RASSF1A), recently cloned from the lung tumor locus at 3p21.3, was shown to be frequently inactivated by hypermethylation of its promoter region in a number of malignancies. We have investigated the expression and epigenetic changes of this novel universal tumor suppressor gene in pituitary adenomas and correlated the data with clinicopathologic findings. Fresh frozen normal pituitary tissues and $\mathbf{5 2}$ primary pituitary adenomas including all major types were examined. Methylation-specific polymerase chain reaction (MSP), combined bisulfite restriction analysis (COBRA), bisulfite sequencing and semiquantitative reverse transcriptionpolymerase chain reaction were used to analyze DNA promoter methylation status and the mRNA expression of RASSF1A, respectively. High levels of RASSF1A transcript and no methylation of the RASSF1A promoter were found in normal pituitary tissues. RASSF1A promoter methylation was detected in 20 of 52 (38\%) adenomas including all major types of pituitary adenomas. However, a lower frequency of methylation of the RASSF1A promoter was found in gonadotroph cell adenomas (15\%) compared with growth hormone cell, prolactin cell, or adrenocorticotropic hormone cell adenomas (54, 46 and $50 \%$, respectively). Methylation frequency was higher in the most aggressive adenomas (87\% in grade IV tumors, $P=0.0163)$. In addition, methylation of the RASSF1A promoter potentially correlated with higher labeling index of the proliferation marker Ki-67 $(P=0.1475)$. Loss or significant reduction of RASSF1A messenger RNA transcripts was identified in 18 of $20(90 \%)$ adenomas with hypermethylation of $\operatorname{RASSF1A}(P<0.0001)$. Our data suggest that promoter hypermethylation of RASSF1A and resultant alterations of RASSF1A expression may play a critical role in pituitary tumorigenesis and may be involved in tumor progression.

Laboratory Investigation (2005) 85, 464-473, advance online publication, 14 February 2005; doi:10.1038/labinvest.3700248
\end{abstract}

Keywords: RASSF1A; tumor suppressor gene; methylation; pituitary adenoma; tumorigenesis

Pituitary adenomas are common and potentially serious neoplasms. They can cause mood disorders, sexual dysfunction, infertility, obesity, visual disturbances, hypertension, diabetes mellitus and

Correspondence: Dr ZR Qian, MD, PhD, Department of Patho$\operatorname{logy} \&$ Satellite Venture Business Laboratory, Institute of Health Biosciences, The University of Tokushima Graduate School, 3-18-15 Kuramoto-cho, 2-1 Minami-josanjima, Tokushima 770-8503, Japan.

E-mail: zrqian@basic.med.tokushima-u.ac.jp

Received 18 November 2004; revised 31 December 2004; accepted 3 January 2005; published online 14 February 2005 accelerated heart disease. Although the majority of tumors are benign, a proportion may invade the surrounding structures such as the sphenoid sinus, the cavernous sinus, and even the brain. The pathogenetic mechanisms underlying pituitary adenoma formation and progression remain unclear. The genetic mutations in classic oncogenes and tumor suppressor genes are rarely found in these tumors. ${ }^{1}$ Progress in understanding the molecular basis of pituitary adenomas might allow the development of early diagnostic markers and novel therapeutic strategies. 
Aberrant methylation of normally unmethylated CpG islands in promoter regions of genes represents an epigenetic change that results in transcriptional inactivation of tumor suppressor genes (TSGs). While genetic events such as mutation, deletion, or rearrangement are well known, aberrant methylation is recognized as an additional mechanism contributing to tumorigenesis. ${ }^{2,3}$

The methylation of CpG islands in several TSGs has been found in virtually every type of human neoplasms ${ }^{2-4}$ including pituitary tumors. ${ }^{5}$ The mechanism of p16 downregulation probably involves cyclin-dependent kinase inhibitor 2A (CDKN2A) methylation in most types of pituitary adenoma, but remain to be determined in growth hormone (GH) cell adenomas; ${ }^{6,7}$ and retinoblastoma 1 (RB1) gene methylation with tumor subtype specificity was described in pituitary tumors. ${ }^{8}$ Loss of Ecadherin associated with methylation of cadherin 1 (CDH1) has been identified in sparsely granulated GH-cell adenomas, ${ }^{9}$ but not in prolactinomas. ${ }^{10}$ Preferential loss of death-associated protein kinase (DAPK) expression in invasive pituitary tumors was reported to be associated with CpG island methylation. ${ }^{11}$ Thus, inactivation of TSGs due to hypermethylation of $\mathrm{CpG}$ islands may play an important role in pituitary tumorigenesis and needs further investigation.

Recently, the Ras association domain family 1 (RASSF1) gene was found as a tumor suppressor gene at 3p21.3 by its frequent epigenetic silencing and loss of heterozygosity (LOH) in lung cancers and lung cancer cell lines. ${ }^{12}$ RASSF 1 encodes more than seven isoforms including two major transcripts, known as $\mathrm{A}$ and $\mathrm{C}$, which are derived from alternative mRNA splicing and use of different promoter sites. Hypermethylation of the RASSF1A promoter has been frequently identified in breast, ovarian, nasopharyngeal, kidney, bladder, gastric, prostate, thyroid, esophageal squamous cell carcinomas, uterine cervical adenocarcinoma and malignant cutaneous melanoma. ${ }^{13-23}$ These findings suggested that RASSF1A may be a universal TSG involving in tumorigenesis.

Furthermore, the ability of RASSF1A to suppress the malignant phenotype has been confirmed both in vitro and in vivo. Re-expression of RASSF1A gene in lung and renal cell carcinoma cell lines lacking endogenous expression of RASSF1A suppressed colony formation, anchorage-independent growth, ${ }^{12,16,24}$ and tumor formation in nude mice. ${ }^{12,24}$ In addition, RASSF1C protein was found to bind Ras in a GTP-dependent manner both in vitro and in vivo and mediate the apoptotic effects of oncogenic Ras. ${ }^{25}$ However, the putative tumor suppressive function of RASSF1C requires much further investigation.

To understand whether the promoter hypermethylation of RASSF1A could play a role in human pituitary tumorigenesis, we examined the frequency of methylation of RASSF1A gene promoter in pituitary adenomas of various types; the expression of the RASSF1A gene was also investigated. In addition, we analyzed the potential clinicopathological implication of hypermethylation of RASSF1A in pituitary adenomas.

\section{Materials and methods}

\section{Human Pituitary Tissues and Adenomas}

Four normal human adenohypophyses were obtained at autopsy from patients with no evidence of endocrine abnormality; they were examined histologically and using immunocytochemistry to exclude the possibility of incidental pathology. A total of 52 pituitary adenoma specimens were obtained at the time of surgery at Tokushima University Hospital (Tokushima, Japan) and Toranomon Hospital (Tokyo, Japan). All samples were frozen and stored at $-70^{\circ} \mathrm{C}$. Tumors were characterized based on the clinical, radiological, histological, and immunohistochemical features (Table 1). ${ }^{26}$ There were 31 clinically functional tumors (13 somatotroph adenomas, one mammosomatotroph adenoma, 11 lactotroph adenomas, four corticotroph adenomas associated with Cushing's disease, two thyrotroph adenomas) and 21 clinically nonfunctioning adenomas (four silent corticotroph adenomas, 13 gonadotroph adenomas, three silent subtype three adenomas, and one null cell adenomas characterized by lack of immunoreactivity for all anterior pituitary hormones). Tumor size and invasiveness were defined on the basis of preoperative radiological investigations and operative findings and with a modified Hardy's classification. ${ }^{27}$ Grade I tumors are microadenomas $(<1 \mathrm{~cm}$ in diameter) and grade II tumors consisted of enclosed macroadenomas ( $\geq 1 \mathrm{~cm}$ in diameter) with or without suprasellar extension. Both grade I and II tumors are defined as noninvasive. Grade III tumors show local invasiveness with evidence of bony destruction and tumor within the sphenoid and/or cavernous sinus. Grade IV tumors demonstrate CNS/extracranial spread with or without metastases. Grade III and IV tumors were considered to be invasive. Thus, 52 tumors included five tumors of grade I, 24 tumors of grade II, 17 tumors of grade III, and six tumors of grade IV (29 noninvasive and 23 invasive adenomas; Table 1).

\section{DNA Isolation and Sodium Bisulfite Modification}

DNA was extracted using the Qiagen DNeasy Tissue Kit (Qiagen, Stanford, CA, USA) according to the manufacturer's protocol. Genomic DNA was modified by sodium bisulfite treatment and purified using the CpGenome DNA Modification Kit (Intergen, Purchase, NY, USA) according to the manufacturer's recommendations. 
Table 1 Summary of RASSF1A methylation in normal pituitary tissues and pituitary adenomas

\begin{tabular}{|c|c|c|c|c|}
\hline \multirow[t]{2}{*}{ Variable } & \multirow[t]{2}{*}{ Case numbers } & \multicolumn{2}{|c|}{ RASSF1A methylation } & \multirow[t]{2}{*}{$\mathrm{P}$} \\
\hline & & + & - & \\
\hline Normal & 4 & 0 & 4 & \\
\hline Patients & 52 & $20(38 \%)$ & $32(62 \%)$ & \\
\hline Age (years) & & $49.2 \pm 4.1$ & $45.1 \pm 2.5$ & 0.469 \\
\hline Gender & & & & 0.0391 \\
\hline Male & 25 & $6(24 \%)$ & $19(76 \%)$ & \\
\hline Female & 27 & $14(52 \%)$ & $11(48 \%)$ & \\
\hline Functional & & & & 0.2276 \\
\hline $\mathrm{GH}$ & 13 & 7 (54\%) & $6(46 \%)$ & \\
\hline GH/PRL & 1 & 0 & $1(100 \%)$ & \\
\hline PRL & 11 & $5(46 \%)$ & $6(54 \%)$ & \\
\hline ACTH & 4 & $2(50 \%)$ & $2(50 \%)$ & \\
\hline $\mathrm{TSH}$ & 2 & 0 & $2(100 \%)$ & \\
\hline Total & 31 & $14(45 \%)$ & $17(55 \%)$ & \\
\hline \multicolumn{5}{|l|}{ Non-functional } \\
\hline ACTHs & 4 & $2(50 \%)$ & $2(50 \%)$ & \\
\hline FSH/LH & 13 & $2(15 \%)$ & $11(85 \%)$ & \\
\hline Subtype 3 & 3 & $2(67 \%)$ & $1(33 \%)$ & \\
\hline Null cell & 1 & 0 & $1(100 \%)$ & \\
\hline Total & 21 & $6(29 \%)$ & $15(71 \%)$ & \\
\hline \multicolumn{5}{|l|}{ Grade } \\
\hline I & 5 & $2(40 \%)$ & $3(60 \%)$ & 0.0163 \\
\hline II & 24 & 8 (33\%) & $16(67 \%)$ & \\
\hline III & 17 & $5(29 \%)$ & $12(71 \%)$ & \\
\hline IV & 6 & $5(83 \%)$ & $1(17 \%)$ & \\
\hline Invasion & & & & 0.5078 \\
\hline Invasive & 23 & $10(44 \%)$ & $13(56 \%)$ & \\
\hline Noninvasive & 29 & $10(35 \%)$ & $19(65 \%)$ & \\
\hline Ki-67 LI (\%) & & $1.58 \pm 0.3$ & $1.15 \pm 0.2$ & 0.1475 \\
\hline$\geq 2.5$ & 12 & $7(\overline{35} \%)$ & $5(1 \overline{6} \%)$ & 0.1067 \\
\hline$<2.5$ & 40 & $13(65 \%)$ & $27(84 \%)$ & \\
\hline
\end{tabular}

Normal, normal pituitary tissue; GH, somatotroph adenoma; GH/PRL, mammosomatotroph adenoma; PRL, lactotroph adenoma; ACTH, corticotroph adenoma; ACTHs, silent corticotroph adenoma; TSH, thyrotroph adenoma; FSH/LH, gonadotroph adenoma; subtype 3, silent subtype 3 adenoma; null cell, null cell adenoma; LI, labeling index.

${ }^{\mathrm{a}}$ Functional vs nonfunctional.

b $\mathrm{I}+\mathrm{II}+\mathrm{III}$ vs IV.

\section{Methylation-Specific PCR}

The promoter methylation status of RASSF1A was investigated by methylation-specific PCR (MSP) assay as described previously. ${ }^{24,28}$ Two sets of RASSF1A promoter-specific primers described by Burbee et $a l^{24}$ were used to specifically amplify methylated and unmethylated DNA sequences. CpGenome $^{\mathrm{TM}}$ Universal Methylated DNA (Intergen) was used as methylated control and bisulfitemodified genomic DNA from HeLa cells served as an unmethylated control. ${ }^{12,20}$ Polymerase chain reaction (PCR) products were separated in $2 \%$ agarose gels and visualized under ultraviolet (UV) illumination. The bisulfite reaction and MSP for all samples were repeated to confirm methylation status. Both methylated and unmethylated PCR products were purified for directly sequencing using the NucleoSpin ${ }^{\circledR}$ Extract Kit (Macherey-nagel, Düren, Germany). Cycle sequencing was performed using the BigDye Terminator V1.1 Cycle sequencing kit (Applied Biosystems, Foster City, CA, USA) and the ABI PRISM 310 Genetic Analyzer (Applied Biosystems).

\section{Combined Bisulfite Restriction Analysis (COBRA) and Bisulfite Sequencing}

The methylation status of the RASSF1A promoter region was also determined by COBRA $^{29}$ and bisulfite genomic sequencing ${ }^{30}$ with primers described by Dammann et al. ${ }^{12,13}$ An amount of $100 \mathrm{ng}$ of bisulfite-treated DNA was amplified with primers MU379 and ML730 in $20 \mu \mathrm{l}$ of reaction buffer containing $200 \mu \mathrm{M}$ of each deoxynucleotide triphosphate and Taq polymerase (AmpliTaq Gold; PE Applied Biosystems) and initial incubation at $95^{\circ} \mathrm{C}$ for $10 \mathrm{~min}$, followed by denaturation at $95^{\circ} \mathrm{C}$ for $15 \mathrm{~s}$, $55^{\circ} \mathrm{C}$ for $15 \mathrm{~s}$, and $74^{\circ} \mathrm{C}$ for $30 \mathrm{~s}$ for 20 cycles. A seminested PCR was performed using $1 \mu \mathrm{l}$ of the initially amplified products, and an internal primer ML561 and the primer MU379 in $50 \mu \mathrm{l}$ of total reaction volume with similar conditions as described for the preceding PCR amplification but for 30 cycles. The PCR products were purified using QIAquick PCR purification kits (Qiagen, Valencia, CA, USA).

For COBRA, $300 \mathrm{ng}$ of the PCR products were digested with $10 \mathrm{U}$ of TaqI (New England Biolab, Beverly, MA, USA) according to the conditions specified by the manufacturer of the enzyme and analyzed on a nondenaturing $6 \%$ polyacrylamide gel, stained with ethidium bromide, and visualized under UV illumination. CpGenome ${ }^{\mathrm{TM}}$ Universal Methylated DNA and DNA from HeLa cells served as positive control and negative control respectively. TaqI digestion of the 205-bp PCR product generates three fragments of 93,81 and $31 \mathrm{bp}$ on complete digestion or partially digested fragments of 174 and $112 \mathrm{bp}$. The density of resultant DNA bands on a gel was estimated by densitometric scanning of ethidium bromide-stained gels and analyzed with computer software (Image, NIH, Bethesda, MD, USA). For each case, the proportion of TaqI resistant (unmethylated) was calculated relative to the PCR product generated in the same reaction that was not subject to restriction digest.

PCR products were sequenced directly to obtain average methylation levels. Further, the PCR products were ligated into the pGEM-T Easy Vector System (Promega) according to the manufacturer's instructions, where six clones were sequenced. All of the described sequences were determined by 
cycle sequencing and run on an automated DNA sequencer to determine the methylation status.

For comparison, we analyzed the $\mathrm{CpG}$ islands in promoter of RASSF1C by COBRA and bisulfite genomic sequencing as described previously. ${ }^{31}$ PCR reactions similar to those described above were performed. For the first PCR reaction, primers M1305 and M1627 were used. For the nested PCR, the internal primers M1318 and M1599 were used. ${ }^{31}$ The analyzed 311-bp promoter fragment contains 38 CpGs, one Sp1 consensus binding site and the putative transcription and translation initiation sites of RASSF1C. This methylated fragment has five $B s t \mathrm{UI}$ sites and digestion results in bands of 140, 89, 31, 21, 16 and $14 \mathrm{bp}$. For COBRA, $300 \mathrm{ng}$ of the PCR products were digested with $10 \mathrm{U}$ of BstUI (New England Biolab) and analyzed on a nondenaturing $6 \%$ polyacrylamide gel. Furthermore, PCR products were subjected for directly sequencing.

\section{Semiquantitative Reverse Transcriptase-Polymerase Chain Reaction Analysis}

Total RNA was extracted using ISOGEN (NIPPON GENE, Toyama, Japan) according to the manufacturer's protocol. Semiquantitative reverse transcriptase-polymerase chain reaction (RT-PCR) analysis was performed as described. ${ }^{17,18}$ The primers for RTPCR were described by Dammann et al. ${ }^{12}$ Briefly, RNA $(0.2 \mu \mathrm{g})$ was preassociated with 50 pmoles of an antisense primer in exon 4. After the RT reaction with StrataScript RT (Stratagene, La Jolla, CA, USA), diluted cDNA (12.5 ng/50 $\mu$ l PCR reaction) was used as template for PCR; 35 cycles were determined to be within the logarithmic phase of amplification and yielded reproducible results with sense primer in exon $2 \alpha \beta$ for RASSF1A or in exon $2 \gamma$ for RASSF1C. RT-PCR for glyceraldehyde-3-phosphate dehydrogenase (GAPDH) served as an internal control using primers described previously. ${ }^{24}$ RT-PCR products $(10 \mu \mathrm{l})$ were resolved on $2 \%$ agarose gels. Quantitation of RASSF1A and $C$ expression levels was achieved by densitometric scanning of ethidium bromide-stained gels. The levels of expression of RASSF1A and $\mathrm{C}$ were analyzed with computer software (Image, NIH) and shown as the ratio of RASSF1A mRNA to GAPDH mRNA or the ratio of $R A S S F 1 C$ mRNA to GAPDH mRNA.

\section{Immunohistochemical Analysis of Ki-67}

Ki-67 antigen immunolocalization based on the labeled streptavidin-biotin method was performed on sections from representative blocks of paraffinembedded tissues used for pathology diagnosis. Ki-67 mouse monoclonal antibody (clone MIB-1, 1:50 dilution; Immunotech, Marseilles, France) was used as in our previous study. ${ }^{10}$ The Ki-67 labeling index (LI) was determined by counting the number of positive cells in a total of 1000 tumor cells observed in several representative high-power fields $(\times 400)$.

\section{Statistical Analyses}

Using StatView J-4.5 software, Mann-Whitney Utest, $\chi^{2}$-test and Spearman's correlation coefficient by rank were performed to determine the significance of associations between different variables. The level of statistical significance was $P<0.05$.

\section{Results}

\section{Methylation Status of RASSF1A in Pituitary Tumors}

At first we used MSP to investigate the epigenetic changes of RASSF1A. We analyzed promoter methylation in four normal pituitary tissues and 52 pituitary adenomas. The methylation region includes $16 \mathrm{CpG}$ sites that were previously found by MSP to be associated with reduced RASSF1A expression $^{24}$ (Figure 1a). Representative results are shown in Figure 1b-d. We then analyzed the relationship between RASSF1A methylation status and the clinicopathological characteristics of 52 patients. The results are summarized in Table 1 . Hypermethylation of the promoter region of RASSF1A was detected in 20 of $52(38 \%)$ pituitary adenomas, but not in four normal pituitary tissues, suggesting that RASSF1A promoter hypermethylation is tumor-specific in the pituitary. Unmethylated bands in MSP were detected in 45 of $52(87 \%)$ adenoma samples and in all four normal tissues. In summary, among 52 pituitary adenomas, seven cases had only methylated alleles, 13 cases had both methylated and unmethylated alleles, and the other 32 cases had only unmethylated alleles as did four normal tissues (Figure 1b, c). Methylated patterns in $R A S S F 1 A$ were found in all major types of pituitary adenomas including seven of $13(54 \%)$ somatotroph adenomas, five of 11 (46\%) lactotroph adenomas, two of four $(50 \%)$ functioning corticotroph adenomas, two of four $(50 \%)$ silent corticotroph adenomas, two of $13(15 \%)$ gonadotroph adenomas, and two of three $(67 \%)$ silent subtype 3 adenomas. The RASSF1A promoter was not methylated in one mammosomatotroph adenoma, two thyrotroph adenomas, and one null cell adenoma. The difference in frequency between functional tumors (14 of 31, $45 \%$ ) and nonfunctional tumors (six of $21,29 \%$ ) was not statistically significant. However, the frequency of RASSF1A methylation in gonadotroph adenomas is noticeably lower than in the other pituitary tumor types.

RASSF1A methylation was detected more in grade IV tumors $(83 \%)$ than in grade I, II, III tumors (40,33, 29\%; respectively) $(P=0.0163)$. In addition, the frequency of RASSF1A methylation was higher in invasive tumors (44\%) than in noninvasive tumors $(35 \%)$, although this trend did not reach 
statistical significance $(P=0.5078)$. The frequency of $R A S S F 1 A$ methylation between female and male was significantly different $(P=0.0391)$. There was no significant correlation between RASSF1A methylation and patient age. The Ki-67 LI was higher in adenomas with $R A S S F 1 A$ methylation $(1.58 \pm 0.3)$ ML730accctcttcctctagcacagtaaagctggcctccagaaacacgggtatctccgcgtggtgcttgcggtcgccgtcgttgtggccg tccggggtggggtgtgaggaggggacgaaggagggaaggaagggcaaggcggggg

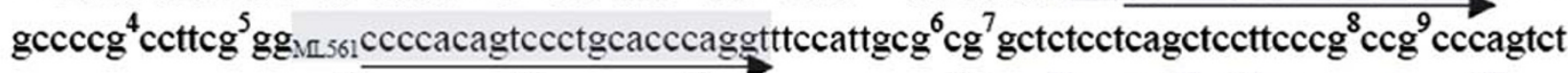
ggatcctgggggaggcg ${ }^{10} \operatorname{ctgaagtcg}^{11} \operatorname{gggcccg}^{12} \operatorname{ccctgtggccccg}^{13} \operatorname{cccg}^{14} \mathrm{gcccg}^{15} \mathrm{cg}^{16} \mathrm{cttgctagc}_{\mathrm{Msp}}{ }^{17} \mathrm{ccc}$ aaagccagcg ${ }^{18}$ aagcacg $^{19}$ ggcccaaccg $^{20}$ ggccatgtcg $^{21}$ ggggagcctgagctcattgagctgcgggagc ${ }_{M U 379}$

b

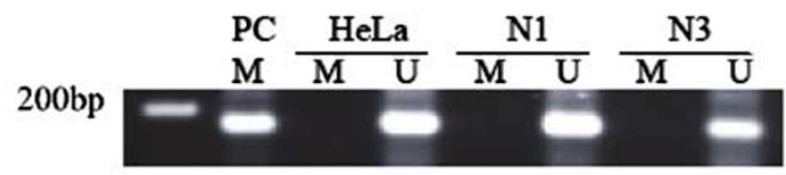

C
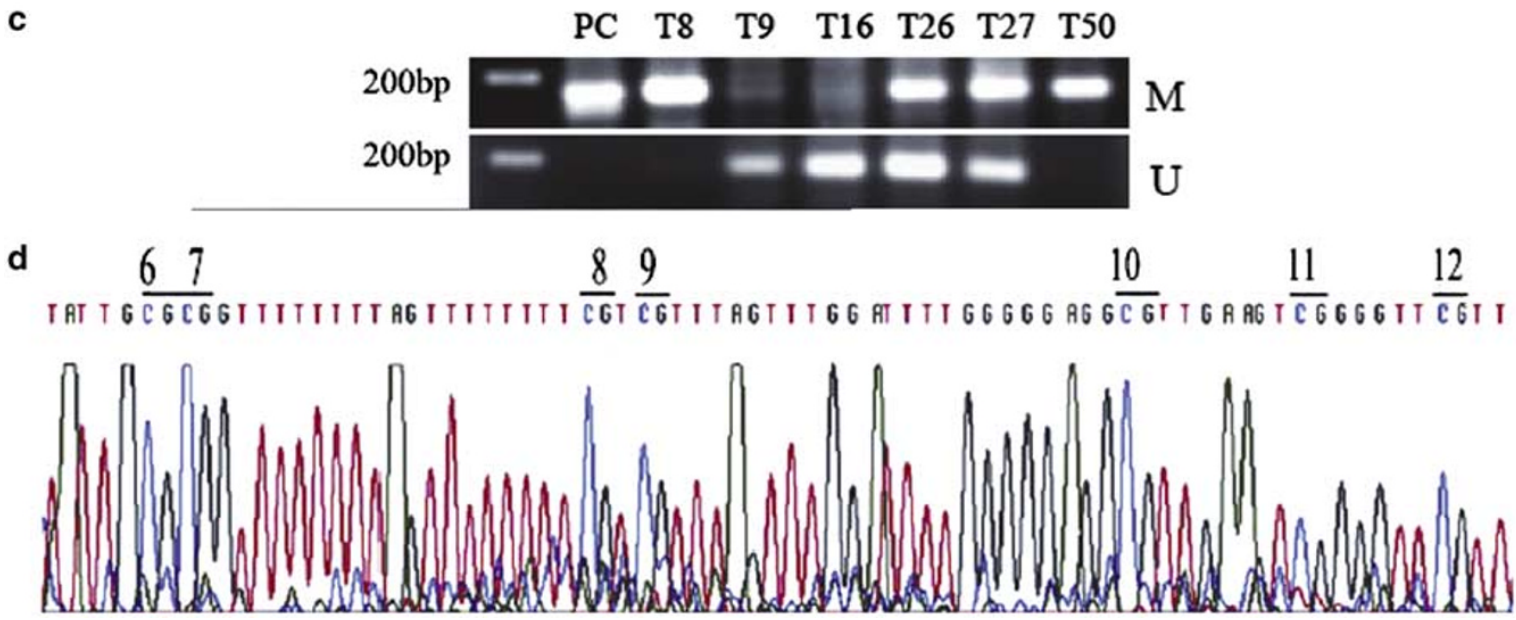

Figure 1 Methylation analysis of RASSF1A promoter in normal pituitary tissues and pituitary tumors. (a) Nucleotide sequence of the CpG island region in the RASSF1A promoter (prior to sodium bisulfite conversion). The 1-16 CpG sites were analyzed by MSP. The 6-21 CpG sites were analyzed by COBRA and bisulfite sequencing. The translational start codon was indicated (arrow). (b) and (c) The methylation status of the RASSF1A promoter was analyzed by MSP in normal pituitary tissues (N1, N3) and pituitary adenomas (T8, T9, T16, T26, T27, and T50). Methylation- (M) and unmethylation-specific (U) primers were used for MSP. The methylation-specific (169 bp) and unmethylation-specific products (169 bp) were resolved on $2 \%$ agarose gel. Universal methylated genomic DNA (PC) and genomic DNA from HeLa cells were used as controls for MSP. (d) Direct sequence of MSP methylation-specific products of RASSF1A from pituitary adenomas. None of the CpG dinucleotides were converted to TpG dinucleotides by bisulfite treatment. The only remaining cytosines in our MSP-amplified DNA segment showing methylated cytosines that are 5' to guanosines are indicated (underlined).

Figure 2 Methylation analysis of $R A S S F 1 A$ and $R A S S F 1 C$ promoter by COBRA and bisulfite sequencing. (a) Methylation analysis of RASSF1A by restriction digestion with TaqI. PCR products from bisulfite-treated DNA obtained from normal samples (N) and primary tumors $(\mathrm{T})$ were digested $(+)$ or mock-digested $(-)$ with TaqI. The molecular sizes are shown on the right in base pairs (bp). T8 and T21 showed total digestion suggesting complete CpG methylation; T26 and T27 showed low proportion of undigested bands (205 bp), and partially digested bands (174 and $112 \mathrm{bp}$ ) in addition to completely digested fragments (93, 81 and $31 \mathrm{bp}$ ). N2 and T16 showed undigestion suggesting unmethylation of CpG islands. (b) Direct sequence of PCR products from bisulfite-treated DNA of pituitary adenomas. The CpG dinucleotides and positions of the TaqI restriction site are underlined. (c) Methylation status of RASSF1A promoter by bisulfite sequencing. The PCR products were subcloned and sequenced. For each case, six independent subclones were represented. O: unmethylated CpG site; $\bullet$ : methylated CpG site. The nucleotide position of each CpG site is indicated at the top. T8, T21, T6 and T27 showed complete or nearly complete methylation in $16 \mathrm{CpG}$ sites. T2 and T16 showed almost complete unmethylation as normal tissues of N2 and N3. (For T8 and T21, MSP detected only methylated band; for T6, T26 and T27, MSP detected both methylated and unmethylated bands; for T2 and T16, MSP detected unmethylated bands as N2 and N3.) (d) Methylation analysis of RASSF1C by restriction digestion with BstUI. PCR products from normal samples (N), primary tumors (T) and universal methylated genomic DNA (PC) were digested (+) or mock-digested (-) with BstUI. The molecular sizes are shown on the right in bp. All the normal and tumor samples were not digested by BstUI, suggesting unmethylation of RASSF1C. 
than in RASSF1A unmethylated adenomas $(1.15 \pm 0.2)$, and higher Ki-67 LI $(\geq 2.5 \%)$ were observed more frequently in $R A S S F 1 A$ methylated adenomas, although these trends did not reach statistical significance $(P=0.1475$ and 0.1067 , respectively).

a

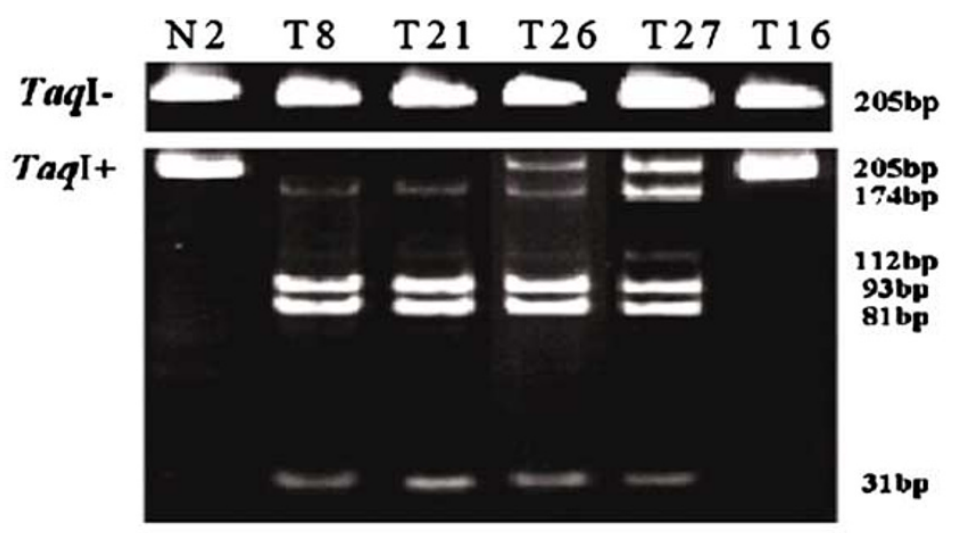

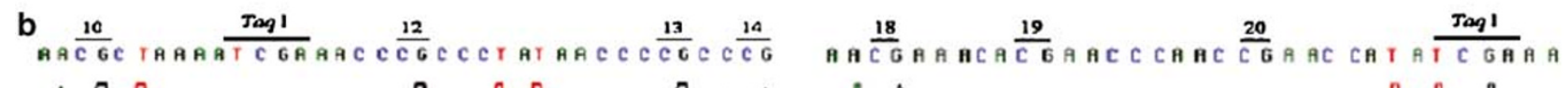
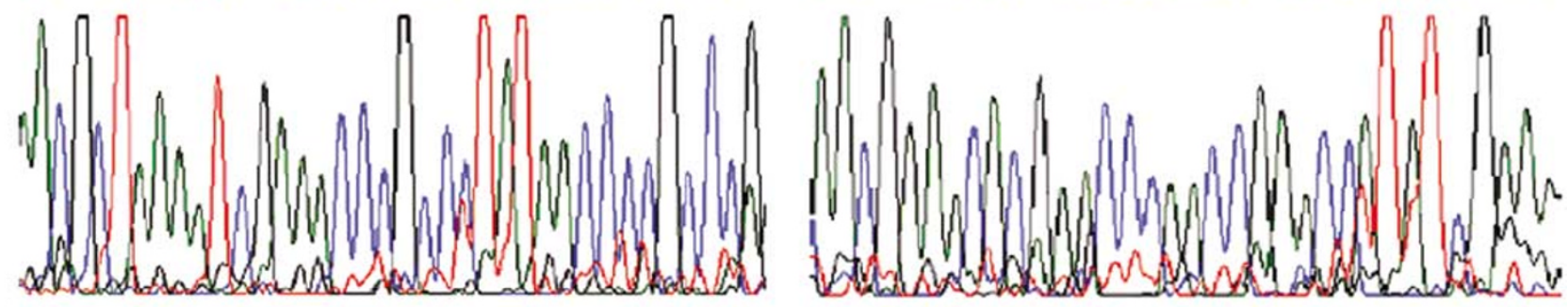

c

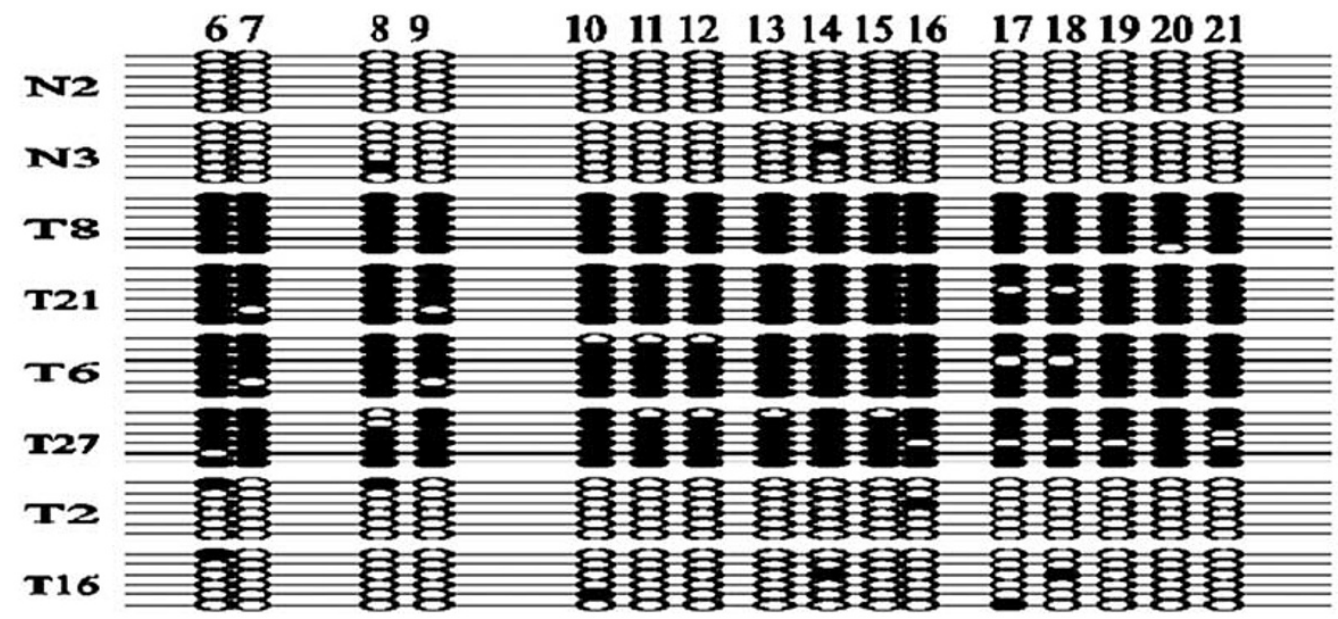

d

N2 N3 T8 T21 T26 T27 PC

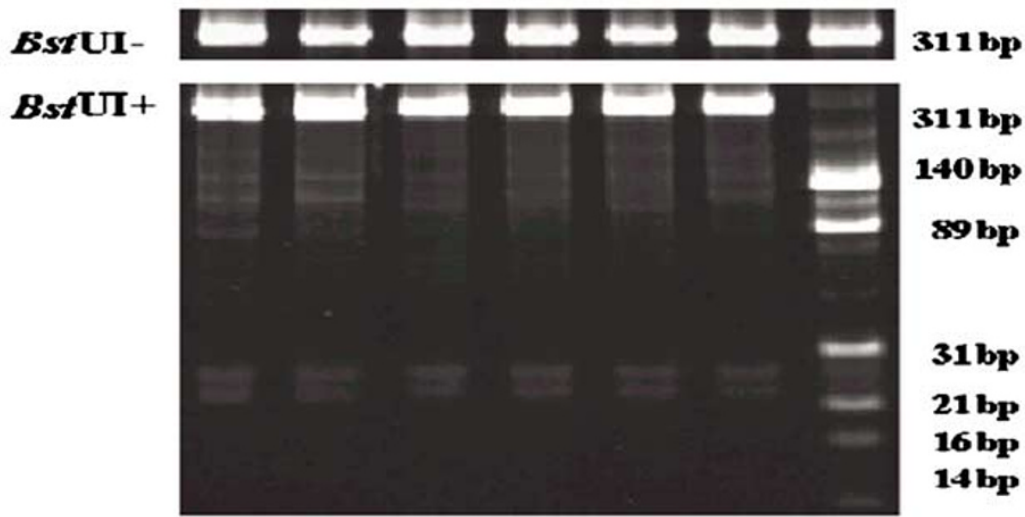


In addition, the specificity of MSP was confirmed by direct sequencing. Six unmethylated MSP products from four normal tissues and two tumors, and 10 methylated MSP products from 10 tumors were directly sequenced. In unmethylated MSP products, all cytosine nucleotides including those in the $\mathrm{CpG}$ islands changed to thymines as a result of bisulfite modification. However, in methylated MSP products, cytosine nucleotides in the $\mathrm{CpG}$ islands remained cytosine (Figure 1d).

\section{Analysis of Methylation Status of RASSF1A and RASSF1C by COBRA and Bisulfite Sequencing}

Since MSP yielded both methylated and unmethylated bands in 13 samples, we further used COBRA and bisulfite sequencing to investigate the methylation status of RASSF1A in four normal tissues and 17 tumors. These 16 CpG sites examined by COBRA and bisulfite sequencing contained $\mathrm{CpG}$ sites from six to 21 in Figure 1. We obtained consistent results by COBRA with TaqI digestion (Figure 2a), bisulfite sequencing (Figure 2b, c) and MSP. COBRA showed positive results in 10 tumors. Four of 10 tumors showed total digestion suggesting complete CpG sites methylation, those tumors with only methylated bands in MSP. Six other tumors that had both methylated and unmethylated alleles by MSP showed a low proportion (3-18\%) of undigested bands, in addition to completely digested bands. Bisulfite direct sequencing also detected a complete or nearly complete $\mathrm{CpG}$ methylation pattern in these 10 tumors. PCR products from four normal pituitary tissues and seven tumors, defined as unmethylated cases by MSP, were not digested by TaqI and also were shown to be unmethylated in all $16 \mathrm{CpG}$ sites by bisulfite direct sequencing. The results of bisulfite direct sequencing were further confirmed by analyzing subclones of PCR products from two normal tissues and six tumors (Figure 2c).

Thus despite a little difference in DNA promoter regions examined and endogenous limitations of each method, the results from these three methods were consistent in this small group.

To prove the specificity of our data showing promoter methylation of $R A S S F 1 A$, we analyzed the methylation status of the promoter of RASSF1C by COBRA and direct sequencing. Four normal pituitary tissues and 52 pituitary adenomas were unmethylated at all $38 \mathrm{CpG}$ sites of RASSF1C (Figure 2d). These results prove that the methylation of CpG islands in RASSF1A promoter is a genespecific event in pituitary adenomas.

\section{Expression of RASSF1A and $C$}

Next we analyzed expression of the RASSF1A gene in 52 pituitary adenomas. For comparison, $R A S S F 1 C$ gene expression was also analyzed. All four normal pituitary tissues as well as HeLa cells expressed easily detectable levels of RASSF1A (RASSF1A/GAPDH, 0.71-0.80, mean 0.75) (Figure 3). Unfortunately, we were not able to obtain matched samples of normal pituitary tissue for each tumor; however, based on this consistent observation, we arbitrarily classified expression levels of
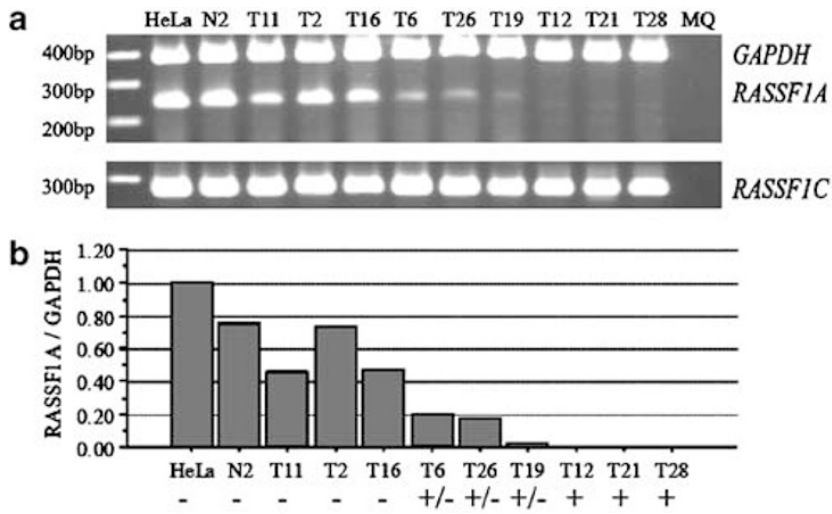

Figure $3 R A S S F 1 A$ and $C$ expression levels in normal pituitary tissues and pituitary tumors. (a) RASSF1A (242 bp) and RASSF1C (272 bp) transcripts were analyzed by semiquantitative RT-PCR using isoform-specific primers. GAPDH expressions were used as endogenous control for RNA integrity and as templates for quantitative analysis. N, normal tissues; T, tumor tissues; MQ, water as a template. (b) Ratios of RASSF1A mRNA to GAPDH mRNA in normal and tumor samples. The methylation status of samples was indicated. + , only methylated alleles; -, only unmethylated alleles; $+/-$, both methylated and unmethylated alleles.

Table 2 Correlation of RASSF1A methylation and expression of $R A S S F 1 A$ in pituitary adenomas

\begin{tabular}{lcccc}
\hline \multirow{2}{*}{$\begin{array}{l}\text { RASSF1A } \\
\text { expression }\end{array}$} & $\begin{array}{c}\text { Case } \\
\text { numbers }\end{array}$ & \multicolumn{2}{c}{ RASSF1A methylation } & \multirow{2}{*}{$\mathrm{P}$} \\
\cline { 3 - 4 } & & + & - & \\
\hline- & 11 & $11(100 \%)$ & 0 & $<0.0001$ \\
$\pm^{\mathrm{a}}$ & 11 & $7(64 \%)$ & $4(36 \%)$ & \\
+ & 30 & $2(7 \%)$ & $28(93 \%)$ & \\
\hline
\end{tabular}

${ }^{\mathrm{a}} \pm$ : significantly lower expression of RASSF1A.

less than one-half of this value, that is, RASSF1A/ $G A P D H,<0.38$ as significant reduction (Figure 3). Loss or significant reduction of RASSF1A expression was found in $42.3 \%(22 / 52)$ of pituitary adenomas (Table 2 and Figure 3). These results demonstrate that loss or significant reduction of RASSF1A expression was frequent in pituitary adenomas.

RASSF1C transcripts were easily detectable in normal pituitary tissues and all pituitary adenomas. None of the tumors exhibited a significantly reduction or overexpression of RASSF1C (Figure 3a), proving that the differential promoter methylation of RASSF $1 A$ and $R A S S F 1 C$ is functionally important in pituitary tumors. 
Correlation between RASSF1A Promoter Hypermethylation and Loss or Reduction of RASSF1A Expression

Four normal pituitary tissues with unmethylated alleles showed high levels of RASSF1A mRNA, comparable to that of HeLa cells. In 28 of 32 unmethylated tumors, RASSF1A mRNA levels were within the normal range. In four unmethylated adenomas, mRNA levels were low. In contrast, RASSF1A mRNA was not detected in 11 tumors with methylated RASSF1A promoters; seven other methylated tumors showed a significant reduction of RASSF1A expression. Just two methylated adenomas showed lower but not significantly reduced mRNA expression. Overall, there was a significant correlation between hypermethylation of RASSF1A promoter and abnormal expression of RASSF1A $(P<0.0001$, Table 2, Figure 3b).

\section{Discussion}

Recently, epigenetic changes in cancer have gained considerable attention as a mechanism of silencing TSGs. ${ }^{32}$ Several major cancers such as lung, breast, colon, and prostate carcinomas have been widely studied for promoter hypermethylation of TSGs. ${ }^{4,33}$ Methylation of the TSGs CDKN2A, RB1, CDH1 and $D A P K$ has been identified in pituitary tumors. ${ }^{6-8,10}$

Hypermethylation of CpG islands in the RASSF1A promoter and its associated gene silencing have been reported in a variety of tumors including endocrine tumors such as small cell lung cancer, thyroid carcinoma and pancreatic endocrine tumors. ${ }^{12,20,34}$ In the current study, we demonstrate RASSF $1 A$ promoter hypermethylation in $38 \%$ of pituitary adenomas including all the major clinically functioning and hormonally inactive types. RASSF1A methylation was significantly associated with loss or reduction of mRNA expression in these pituitary adenomas. Noticeable, RASSF1A methylation was detected in 11 tumors which exhibited no RASSF1A expression and in other seven tumors which showed significant reduction of RASSF1A expression. In contrast, the RASSF1A promoter was unmethylated and mRNA levels were high in normal pituitary tissues. As, there is no working antibody, we could not further analyze the relationship between methylation status and protein expression of RASSF1A. Nevertheless, our findings indicate that epigenetic inactivation of $R A S S F 1 A$ is associated with pituitary tumorigenesis.

There was no significant correlation between RASSF1A methylation and patient age, thus methylation of RASSF1A in pituitary adenomas is not attributable to the aging process, as has been described for other genes. ${ }^{35}$ Although methylated $R A S S F 1 A$ in tumors was more frequently detected in women than in men, the reason for this genderdependent difference is unclear and requires confirmation in larger numbers of cases. The frequency of RASSF1A methylation was not significantly different between functional and nonfunctional groups. However, it is interesting to note that gonadotroph adenomas show a lower frequency of RASSF1A methylation (15\%) compared to the other three major types: somatotroph, lactotroph and corticotroph adenomas $(54,46$ and $50 \%$; respectively). This tumor type-specific alteration, which is similar to that reported for $C D K N 2 A, R B 1$ and $C D H 1$, indicates that different types of pituitary adenomas may have distinct etiologic factors and pathogenetic mechanisms.

Previous studies attested to an association of RASSF1A methylation with tumor aggressiveness, poor prognosis or tumor stage in several tumor types including bladder cancer, esophageal squamous cell carcinoma, and stage I lung adenocarcinoma. ${ }^{21,36,37}$ We demonstrate here that RASSF1A methylation is detected in all grades of pituitary adenoma and lacks a strict correlation with tumor invasiveness; nevertheless, hypermethylation of RASSF1A occurred more frequently in grade IV adenomas $(83 \%)$ that exhibit a more aggressive behavior. These findings suggest that in addition to a role in tumorigenesis, RASSF1A methylation may also regulate the progression of pituitary adenoma. However, the numbers of grade I and IV tumors in this series is small, and further investigation of larger series is required.

The precise mechanism of the tumor-suppressing activity of RASSF1A is still unclear, and more biochemical and genetic data are needed to understand its role in tumorigenesis. Recently, Shivakumar et $a l^{38}$ reported that $R A S S F 1 A$ can inhibit cell proliferation and block cell cycle progression by preventing cyclin D1 accumulation. Furthermore, Song et $a 1^{39}$ reported that RASSF1A regulates mitosis by inhibiting the APC-Cdc20 complex during prometaphase. Thus we analyzed the relation between RASSF1A methylation and proliferation of pituitary adenomas. Methylated tumors had a higher Ki-67 LI than unmethylated tumors. In addition, a higher Ki-67 LI was more frequently observed in methylated tumors than unmethylated tumors, although these differences did not reach statistical significance. It will be interesting to widely investigate this phenomenon in other tumors.

$R A S S F 1 C$ is another main isoform of RASSF1. In the present study, RASSF1C did not show hypermethylation and its mRNA was detected in similar amounts in all samples analyzed. Our results are same as those found in lung and breast tumors. ${ }^{12,24}$ However, a recent study demonstrated that RASSF1C also functions as a TSG, inducing growth inhibition in a prostate cell line LNCaP, a renal cell carcinoma line KRC/Y and in SCID mice; moreover, mutation or loss of expression of RASSF1C is associated with increased tumor growth in vivo. In addition, $R A S S F 1 C$ was shown to induce cell cycle arrest in KRC/Y cells. ${ }^{40}$ These findings indicate that 
$R A S S F 1 C$ may serve as a tumor suppressor gene and could have tissue-specific effects. Our data provide evidence that the methylation of the RASSF1A promoter is a specific and functionally important event in the expression of this TSG that is not globally related to other TSGs, including its closely situated homologue RASSF1C.

For altered expression of a gene, both alleles of a TSG need to be inactivated in the classical and revised two-hit hypothesis. ${ }^{2,41} R A S S F 1 A$ inactivation accomplished via hypermethylation has been observed in many different kinds of cancers with frequent allelic imbalance at $3 p 21.3$. In some of them, RASSF1A promoter methylation has been associated with $\mathrm{LOH},{ }^{36,42}$ but it has been found less frequently in other types of cancer. ${ }^{14}$ Most recently, methylation of $R A S S F 1 A$ has been identified in malignant cutaneous melanoma, medulloblastoma, and hepatocellular carcinoma that have rare $\mathrm{LOH}$ at 3p21.3. ${ }^{22,43,44}$ In addition, mutational inactivation of this gene is very rare. ${ }^{12}$ These data suggested that promoter hypermethylation is the major mechanism for inactivation of RASSF1A in human tumors. Thus, it is not surprising that RASSF1A methylation has been identified in a considerable proportion of pituitary adenomas and correlated with loss or reduction of mRNA expression. In this study, MSP detected only unmethylated alleles in seven tumors and these tumors showed completely undetectable mRNA expression. Furthermore COBRA and bisulfite sequencing confirmed that there were only methylated alleles in these tumors. Owing to the rarity of $\mathrm{LOH}$ at $3 \mathrm{p} 21.3$ in pituitary adenomas, ${ }^{45}$ RASSF1A inactivation caused by methylation of both alleles may be a critical event in pituitary tumorigenesis. In addition, 11 tumors had absent or significantly reduced $R A S S F 1 A$ expression despite the presence of both methylated and unmethylated alleles. A variety of possibilities may explain these data. First, it is possible that RASSF1A may belong to the group of haploinsufficient tumor suppressor genes that promote tumor formation through the inactivation of only one allele. ${ }^{46}$ Alternatively, silencing could be due to other mechanisms, such as a separate genetic lesion to inactivate an unmethylated allele ${ }^{20}$ or lack of transactivation factors. Another possibility is that nontumorous cells contaminating the frozen sample may contribute to the presence of unmethylated bands in MSP and detectable levels of mRNA. This possibility must be considered since MSP analysis is a very sensitive method that can detect a methylated gene at $0.1 \%$ dilution. ${ }^{28}$ Therefore, unmethylated genes in nontumorous cells can be easily detected. Owing to our COBRA and bisulfite sequencing results, nontumorous cells contaminating the frozen sample may be the main reason for the unmethylated bands detected by MSP in these tumors. Also, bisulfite sequencing data showing complete or nearly complete methylation in all CpG islands, excluding the possibility that partial methylation could cause gene expression in the tumor cells despite hypermethylation pattern in MSP. ${ }^{47,48}$

The results presented here suggest that promoter hypermethylation of the RASSF1A gene has a critical role in pituitary tumor pathogenesis. The $R A S S F 1 A$ gene probably has a fundamental role as a tumor suppressor gene at an early stage of pituitary tumorigenesis and also may be involved in tumor proliferation and aggressiveness.

\section{Acknowledgements}

We thank $\mathrm{H}$ Siomi Lab, institute for genome research, for excellent technical assistance.

\section{References}

1 Asa SL, Ezzat S. The pathogenesis of pituitary tumors. Nat Rev Cancer 2002;2:836-849.

2 Jones PA, Laird PW. Cancer epigenetics comes of age. Nat Genet 1999;21:163-167.

3 Plass C. Cancer epigenomics. Hum Mol Genet 2002; 11:2479-2488.

4 Jones PA, Baylin SB. The fundamental role of epigenetic events in cancer. Nat Rev Genet 2002;3:415-428.

5 Farrell WE, Clayton RN. Epigenetic change in pituitary tumorigenesis. Endocr Relat Cancer 2003;10:323-330.

6 Seemann N, Kuhn D, Wrocklage C, et al. CDKN2A/P16 inactivation is related to pituitary adenoma type and size. J Pathol 2001;193:491-497.

7 Simpson DJ, McNicol AM, Murray DC, et al. Molecular pathology shows p16 methylation in nonadenomatous pituitaries from patients with Cushing's disease. Clin Cancer Res 2004;10:1780-1788.

8 Simpson DJ, Hibberts NA, McNicol AM, et al. Loss of $\mathrm{pRb}$ expression in pituitary adenomas is associated with methylation of the RB1 CpG island. Cancer Res 2000;60:1211-1216.

$9 \mathrm{Xu} \mathrm{B}$, Sano T, Yoshimoto K, et al. Downregulation of E-cadherin and its undercoat proteins in pituitary growth hormone cell adenomas with prominent fibrous bodies. Endocr Pathol 2002;13:341-351.

10 Qian ZR, Li CC, Yamasaki H, et al. Role of E-cadherin, alpha-, beta-, and gamma-catenins, and p120 (cell adhesion molecules) in prolactinoma behavior. Mod Pathol 2002;15:1357-1365.

11 Simpson DJ, Clayton RN, Farrell WE. Preferential loss of death associated protein kinase expression in invasive pituitary tumours is associated with either CpG island methylation or homozygous deletion. Oncogene 2002;21:1217-1224.

12 Dammann R, Li C, Yoon JH, et al. Epigenetic inactivation of a RAS association domain family protein from the lung tumour suppressor locus 3p21.3. Nat Genet 2000;25:315-319.

13 Dammann R, Yang G, Pfeifer GP. Hypermethylation of the $\mathrm{CpG}$ island of Ras association domain family $1 \mathrm{~A}$ (RASSF1A), a putative tumor suppressor gene from the 3p21.3 locus, occurs in a large percentage of human breast cancers. Cancer Res 2000;61:3105-3109.

14 Agathanggelou A, Honorio S, Macartney DP, et al. Methylation associated inactivation of RASSF1A from 
region 3p21.3 in lung, breast and ovarian tumours. Oncogene 2001;20:1509-1518.

15 Lo KW, Kwong J, Hui AB, et al. High frequency of promoter hypermethylation of RASSF1A in nasopharyngeal carcinoma. Cancer Res 2001;61:3877-3881.

16 Dreijerink K, Braga E, Kuzmin I, et al. The candidate tumor suppressor gene, RASSF1A, from human chromosome 3p21.3 is involved in kidney tumorigenesis. Proc Natl Acad Sci USA 2001;98:7504-7509.

17 Lee MG, Kim HY, Byun DS, et al. Frequent epigenetic inactivation of RASSF1A in human bladder carcinoma. Cancer Res 2001;61:6688-6692.

18 Byun DS, Lee MG, Chae KS, et al. Frequent epigenetic inactivation of RASSF1A by aberrant promoter hypermethylation in human gastric adenocarcinoma. Cancer Res 2001;61:7034-7038.

19 Kuzmin I, Gillespie JW, Protopopov A, et al. The RASSF1A tumor suppressor gene is inactivated in prostate tumors and suppresses growth of prostate carcinoma cells. Cancer Res 2002;62:3498-3502.

20 Schagdarsurengin U, Gimm O, Hoang-Vu C, et al. Frequent epigenetic silencing of the $\mathrm{CpG}$ island promoter of RASSF1A in thyroid carcinoma. Cancer Res 2002;62:3698-3701.

21 Kuroki T, Trapasso F, Yendamuri S, et al. Promoter hypermethylation of RASSF1A in esophageal squamous cell carcinoma. Clin Cancer Res 2003;9:1441-1445.

22 Cohen Y, Singer G, Lavie O, et al. The RASSF1A tumor suppressor gene is commonly inactivated in adenocarcinoma of the uterine cervix. Clin Cancer Res 2003;9:2981-2984.

23 Spugnardi M, Tommasi S, Dammann R, et al. Epigenetic inactivation of RAS association domain family protein 1 (RASSF1A) in malignant cutaneous melanoma. Cancer Res 2003;63:1639-1643.

24 Burbee DG, Forgacs E, Zochbauer-Muller S, et al. Epigenetic inactivation of RASSF1A in lung and breast cancers and malignant phenotype suppression. J Nat Cancer Inst 2001;93:691-699.

25 Vos MD, Ellis CA, Bell A, et al. Ras uses the novel tumor suppressor RASSF1 as an effector to mediate apoptosis. J Biol Chem 2000;275:35669-35672.

26 Asa SL. Tumors of the Pituitary Gland. Atlas of Tumor Pathology, Series Fascicle 22. Armed Forces Institute of Pathology: Washington, DC, USA, 1998, pp 49-54.

27 Hardy J. Transsphenoidal microsurgical treatment of pituitary tumours. In: Linfoot J (ed.) Recent Advances in the Diagnosis and Treatment of Pituitary Tumours. Raven Press: New York, 1979, pp 375-388.

28 Herman JG, Graff JR, Myohanen S, et al. Methylationspecific PCR: a novel PCR assay for methylation status of CpG islands. Proc Natl Acad Sci USA 1996; 93:9821-9826.

29 Xiong Z, Laird PW. COBRA: a sensitive and quantitative DNA methylation assay. Nucleic Acids Res 1997;15:2532-2534.

30 Frommer M, McDonald LE, Millar DS, et al. A genomic sequencing protocol that yields a positive display of 5-methylcytosine residues in individual DNA strands. Proc Natl Acad Sci USA 1992;89:1827-1831.

31 Dammann R, Takahashi T, Pfeifer GP, et al. The CpG island of the novel tumor suppressor gene RASSF1A is intensely methylated in primary small cell lung carcinomas. Oncogene 2001;20:3563-3567.

32 Herman JG, Baylin SB. Gene silencing in cancer in association with promoter hypermethylation. N Engl J Med 2003;349:2042-2054.

33 Sidransky D. Emerging molecular markers of cancer. Nat Rev Cancer 2002;2:210-219.

34 Dammann R, Schagdarsurengin U, Liu L, et al. Frequent RASSF1A promoter hypermethylation and K-ras mutations in pancreatic carcinoma. Oncogene 2003;22:3806-3812.

35 Ahuja N, Li Q, Mohan AL, et al. Aging and DNA methylation in colorectal mucosa and cancer. Cancer Res 1998;58:5489-5494.

36 Maruyama R, Toyooka S, Toyooka KO, et al. Aberrant promoter methylation profile of bladder cancer and its relationship to clinicopathological features. Cancer Res 2001;61:8659-8663.

37 Tomizawa Y, Kohno T, Kondo H, et al. Clinicopathological significance of epigenetic inactivation of RASSF1A at 3p21.3 in stage I lung adenocarcinoma. Clin Cancer Res 2002;8:2362-2368.

38 Shivakumar L, Minna J, Sakamaki T, et al. The RASSF1A tumor suppressor blocks cell cycle progression and inhibits cyclin D1 accumulation. Mol Cell Biol 2002;22:4309-4318.

39 Song MS, Song SJ, Ayad NG, et al. The tumour suppressor RASSF1A regulates mitosis by inhibiting the APC-Cdc20 complex. Nat Cell Biol 2004;6: 129-137.

$40 \mathrm{Li} \mathrm{J}$, Wang $\mathrm{F}$, Protopopov $\mathrm{A}$, et al. Inactivation of RASSF1C during in vivo tumor growth identifies it as a tumor suppressor gene. Oncogene 2004;23:5941-5949.

41 Knudson AG. Two genetic hits (more or less) to cancer. Nat Rev Cancer 2001;1:157-162.

42 Yu MY, Tong JH, Chan PK, et al. Hypermethylation of the tumor suppressor gene RASSF1A and frequent concomitant loss of heterozygosity at 3 p21 in cervical cancers. Int J Cancer 2003;105:204-209.

43 Lusher ME, Lindsey JC, Latif F, et al. Biallelic epigenetic inactivation of the RASSF1A tumor suppressor gene in medulloblastoma development. Cancer Res 2002;62:5906-5911.

44 Zhong S, Yeo W, Tang MW, et al. Intensive hypermethylation of the CpG island of Ras association domain family $1 \mathrm{~A}$ in hepatitis $\mathrm{B}$ virus-associated hepatocellular carcinomas. Clin Cancer Res 2003;9: 3376-3382.

45 Bates AS, Farrell WE, Bicknell EJ, et al. Allelic deletion in pituitary adenomas reflects aggressive biological activity and has potential value as a prognostic marker. J Clin Endocrinol Metab 1997; 82:818-824.

46 Bai F, Pei XH, Godfrey VL, et al. Haploinsufficiency of p18(INK4c) sensitizes mice to carcinogen-induced tumorigenesis. Mol Cell Biol 2003;23:1269-1277.

47 Foster, SA, Wong DJ, Barrett MT, et al. Inactivation of p16 in human mammary epithelial cells by CpG island methylation. Mol Cell Biol 1998;18:1793-1801.

48 Shim Y Hee, Kang GH, Ro JY. Correlation of p16 hypermethylation with p16 protein loss in sporadic gastric carcinomas. Lab Invest 2000;80:689-695. 